\title{
New design of multi-band negative-index metamaterial and absorber at visible frequencies
}

\author{
Boyi Gong*, Fan Guo, Wenkang Zou and Lin Chen \\ Institute of Fluid Physics, China Academy of Engineering Physics, \\ Mianyang 621999, China \\ *bygong163@163.com \\ Kun Song and Xiaopeng Zhao \\ Department of Applied Physics, Northwestern Polytechnical University, \\ $X i$ 'an 710129, China
}

Received 17 May 2016

Accepted 13 July 2017

Published 31 July 2017

\begin{abstract}
A new negative-index metamaterial (NIM) structure is proposed by designing the metallic holes of traditional double-fishnet (DF) structures from uniform sizes to several different sizes. Numerical results demonstrate that the new metamaterial, as an improved variant of the DF structure, achieved a multi-band negative refractive index across a wide range of visible frequencies from $470 \mathrm{THz}$ to $540 \mathrm{THz}$, which covers the red, orange, yellow, and green regions of the visible spectra. Meanwhile, a low-profile nanostrctured absorber was obtained when one side of the perforated metal layer of this multi-band NIM was substituted with a continuous metal film with the same thickness. The absorber showed the high absorption of more than $95 \%$ at multiple frequencies of $511,520,523$, 525 , and $527 \mathrm{THz}$. The behavior of multi-frequency response effectively broadened the working bandwidth. Finally, the physical mechanism of the multi-band operating characteristics of NIM and absorber was analyzed with the distributions of current intensity at different resonant frequencies.
\end{abstract}

Keywords: Negative-index metamaterial; absorber; visible frequencies; multiple frequencies.

\section{Introduction}

Negative-index metamaterials (NIMs), as artificially structured composites, have been well-established since the appearance of the first NIM in 2001. 1 NIMs exhibit unprecedented properties such as negative refraction, $\frac{2}{2}$ superlens with

This is an Open Access article published by World Scientific Publishing Company. It is distributed under the terms of the Creative Commons Attribution 4.0 (CC-BY) License. Further distribution of this work is permitted, provided the original work is properly cited. 


\section{B. Gong et al.}

sub-wavelength resolution, $\frac{3}{-}$ perfect absorbers, $\frac{4}{,}$ inversed Doppler effect, $\frac{5}{-}$ electromagnetic cloak,,$\frac{6}{6}$ and other characteristics, ${ }^{7-9}$ which are unattainable from naturally occurring materials. Hence, NIMs with reasonable pre-designed properties can be considerably applied in physics, materials, and engineering. Since the first development of NIMs in the microwave range, the artificial magnetism and negative refractive index of these artificial materials have been shifted from the gigahertz frequencies to optical frequencies over the past decade. ${ }^{10}-14$ Numerous NIMs have been designed to achieve negative refractive index because of simultaneous negative values of permittivity $\varepsilon$ and permeability $\mu$ in the same frequency region, which originate from electrical and magnetic resonances of the interior structural units, respectively. However, these characteristics often lead to a narrow operating band in the vicinity of the resonant frequency, thereby seriously impeding the effective application of NIMs. Thus, the working band of NIMs should be broadened to better embody the fascinating properties as mentioned above. Several designs of NIMs with multiple and wide bands have been presented. $\underline{15}-19$ Most of these designs include two or three operating frequencies, which increase the bandwidth to a certain extent and provide some referential methods for designing multi-band metamaterials. However, the frequency range should be extended continuously for practical applications.

In this paper, a multi-band visible NIM has been designed based on doublefishnet (DF) metamaterials which have been extensively investigated as a representative structure of optical NIMs and are composed of two perforated metal films separated by a thin layer of dielectric. ${ }^{20-22}$ In this study, some geometrical structures were modified. The metallic holes, which were arrayed periodically in a two-dimensional (2D) plane, were transformed from having the same sizes into 16 different sizes. The structural parameters were also properly modulated and optimized. Consequently, more than four electromagnetic resonances occurred at a broad optical range, and the combination of these resonances could effectively widen the operating band of negative-index responses. Moreover, a low-profile nanostructured absorber was obtained when one layer of perforated metal was replaced with a continuous metal film with the same thickness. High absorptivity of more than $95 \%$ was achieved around several neighboring visible frequencies. The operating band of the new NIM and absorber contained more responsive frequencies compared with some previous multi-band metamaterial structures. This characteristic would be more favorable for practical applications.

\section{Numerical Simulations}

Our model consists of a metal-dielectric-metal nanostructured stack and has two symmetrical axes in the $x-y$ plane, which are denoted with white lines in Fig. 1. The array of metallic holes is periodically repeated in the $2 \mathrm{D}$ plane by setting the boundary conditions of an electric wall in the $x$ (or $y$ ) direction and a magnetic wall in the $y$ (or $x$ ) direction. The electromagnetic wave is incident normal to the surface 


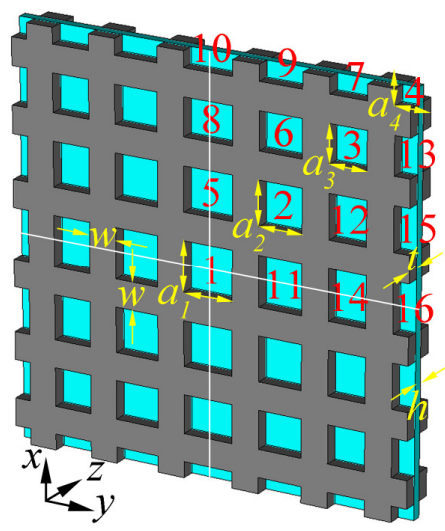

Fig. 1. (Color online) The model of multi-band visible NIM and its geometric dimensions. The upper and lower layers of silver have the same thickness of $t=45 \mathrm{~nm}$, and the PVA layer has a thickness of $h=30 \mathrm{~nm}$. The wall of all holes has a width $w=90 \mathrm{~nm}$. The holes with numbers $1,2,3$ and 4 are squares with lengths of $a_{1}=165 \mathrm{~nm}, a_{2}=145 \mathrm{~nm}, a_{3}=125 \mathrm{~nm}$, and $a_{4}=$ $105 \mathrm{~nm}$, respectively. The other holes are rectangles, and their sizes can be described with $a_{1}, a_{2}, a_{3}$ and $a_{4}$.

(along the $z$ direction). Numerical simulations have been performed with a threedimensional (3D) electromagnetic simulation software based on the finite-difference time-domain method. Silver is selected as the metallic material because of its low Ohmic loss at optical frequencies caused by its small skin depth. This metal starts to strongly deviate from being an ideal conductor in the optical range. The dielectric function of silver should be described by Drude medium model with a plasma frequency of $\omega_{\mathrm{p}}=1.37 \times 10^{16} \mathrm{~Hz}$ and a collision frequency of $\omega_{\mathrm{c}}=8.5 \times 10^{13} \mathrm{~Hz} \cdot{ }^{23}$ The middle dielectric layer as a spacer is polyvinyl alcohol (PVA) with permittivity of $\varepsilon=2.25$ and a thickness of $h=30 \mathrm{~nm}$. All walls of the holes have the same sizes with a width of $w=90 \mathrm{~nm}$ and a thickness of $t=45 \mathrm{~nm}$. A total of 16 different holes are tested and labeled from number 1 to 16 . Holes 1, 2, 3 and 4 are squares with lengths of $a_{1}=165 \mathrm{~nm}, a_{2}=145 \mathrm{~nm}, a_{3}=125 \mathrm{~nm}$, and $a_{4}=105 \mathrm{~nm}$, respectively. The other holes are rectangles, and their sizes can be expressed with $a_{1}, a_{2}, a_{3}$ and $a_{4}$. The lattice constant along the $z$-direction is much smaller than the visible wavelengths. This property can satisfy the requirement of effective medium theory, which stipulates that the interior elementary dimensions along the propagation direction of the incident wave should be on the sub-wavelength scale. ${ }^{24}$

\section{Results and Analysis}

\subsection{Retrieval of effective medium parameters}

The scattering (S) parameters, namely, the magnitude and phase of transmission (S21) and reflection (S11), which are gained from simulations, are shown in Fig. 2. The solid curves represent the coefficients of S21, and dashed curves depict those 

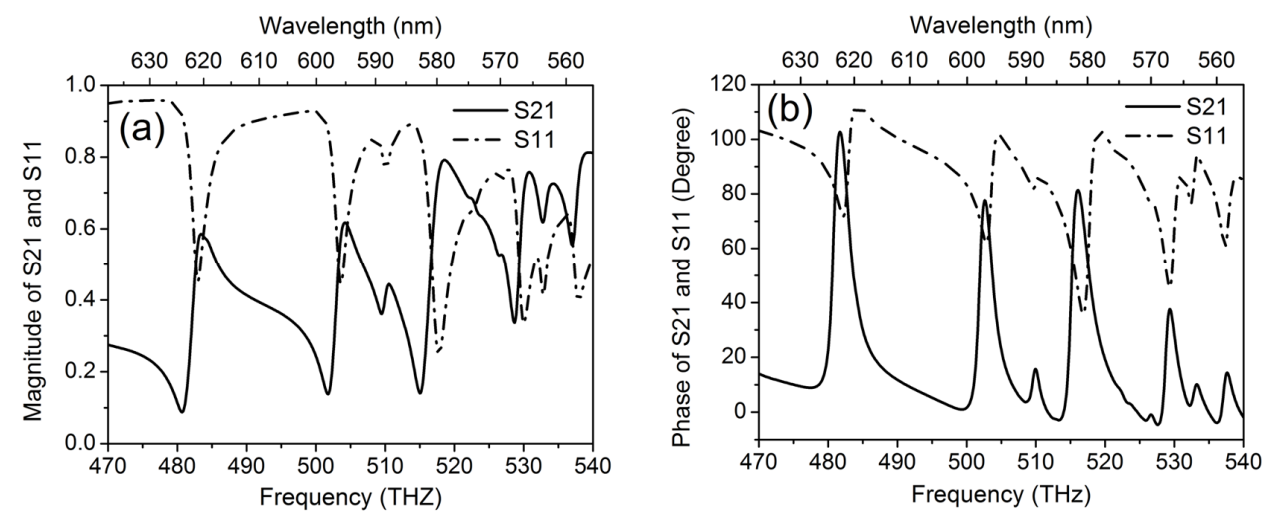

Fig. 2. Scattering parameters of transmission (S21) and reflectance (S11) were obtained from simulations, which are plotted with solid and dashed curves, respectively. (a) Magnitude of S21 and S11. (b) Phase of S21 and S11.

of S11. In Fig. 2(a), it can be seen that the transmission mainly has five local minimum values at 481, 502, 515, 529, and 537 THz. Meanwhile, several phase jumps of transmission at these corresponding frequencies, as observed in Fig. 2(b). Thus, the appearance of transmission values and phase jumps of transmission at the same frequencies obviously indicates the occurrence of electromagnetic resonances at these points.

The effective constitutive parameters, such as permeability $\mu$, permittivity $\varepsilon$, refractive index $n$, and impedance $\mathrm{Z}$ of this new metamaterial can be extracted by a standard retrieval algorithm. ${ }^{25}$ The corresponding results are displayed in Figs. 3(a)-3(d), respectively. Several magnetic resonances emerged in Fig. 3(a), which correspond to the positions of the phase jumps of transmission in Fig. $\overline{2}$ (b). However, only four resonances are strong enough to realize $\mu<0$ (if this is not a special station, then it refers to the real parts in our paper). In Fig. 3(b), the $\varepsilon$ value is kept negative in a wide frequency range, which covers the region of $\mu<0$. Hence, a background of $\varepsilon<0$ is established for magnetic resonances, and the overlap range of $\mu<0$ and $\varepsilon<0$ leads to the negative index $(n<0)$, as shown in Fig. 3(c). The multiple bands of $n<0$ cross a very wide range of $470-540 \mathrm{THz}$, which covers the red, orange, yellow, and green regions in the visible spectra. Thus, the wide multiple band of $n<0$ could effectively broaden the working frequency range. In addition, $\mathrm{Z}$, which is also depicted in Fig. 3(d), has a value close to 1 at the frequencies of $n<0$, thereby indicating an approximate match with air.

\subsection{Analysis of physical mechanism}

Physical mechanism is explored with resonance characteristics to explain the reason for such a wide multi-band negative-index property. Figures 4(a)-4(d), respectively exhibit the intensity distributions of resonant currents in the upper and lower silver strips at 481, 502, 515, and $529 \mathrm{THz}$, which correspond to the four stronger 

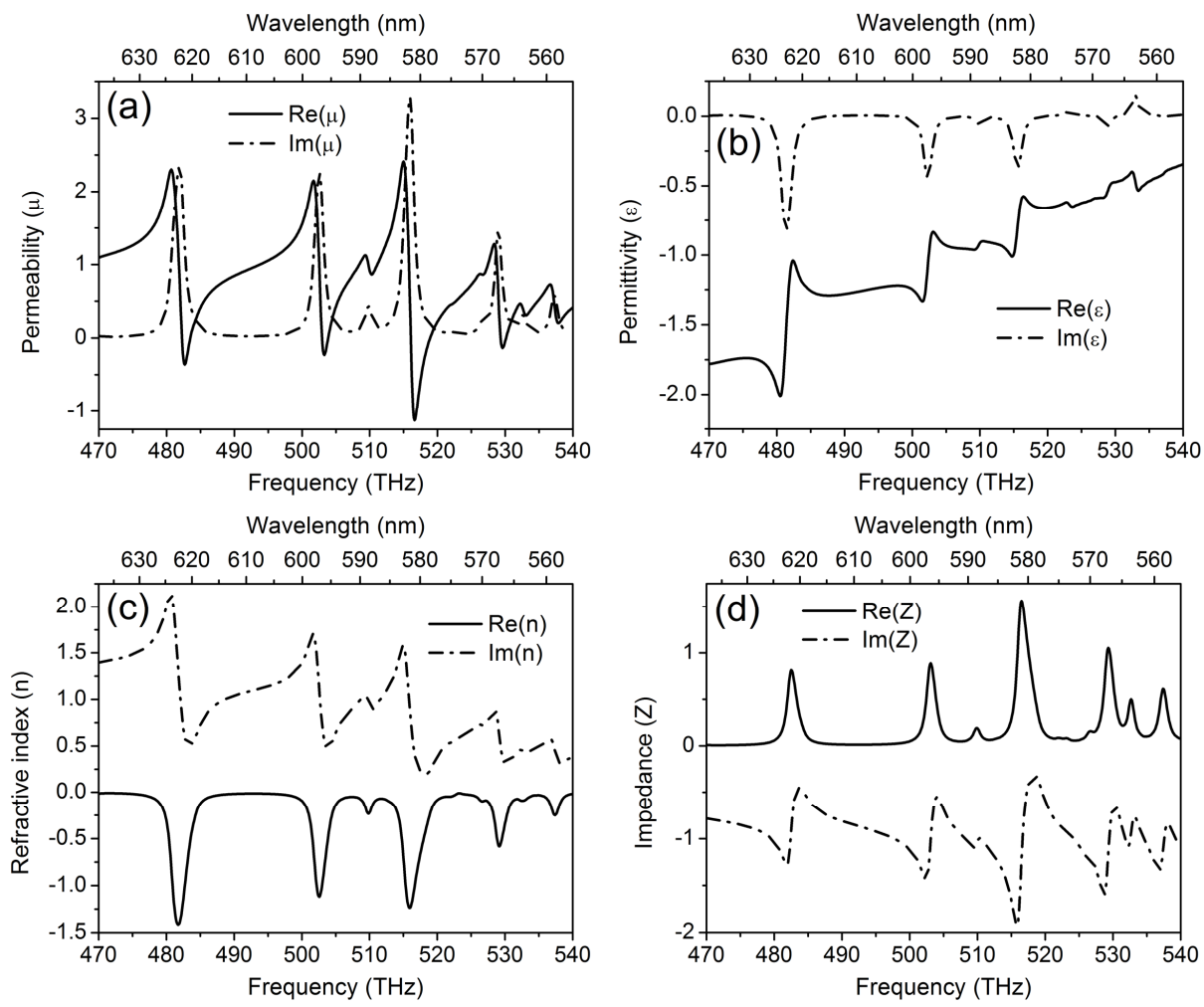

Fig. 3. Spectra of effective medium parameters that were restored from S parameters. Solid curves denote the real parts, and dashed curves indicate the imaginary parts. (a) Permeability $\mu$. (b) Permittivity $\varepsilon$. (c) Refractive index $n$. (d) Impedance Z.

resonances with $n<0$ in Fig. 3(a). The following conclusions could be drawn from the comparative results of Figs. 3 and 4. First, the applied electric field parallel to both strips causes parallel currents in both strips. By contrast, the magnetic field perpendicular to the plane of the strips induces antiparallel currents, thereby resulting in the formation of magnetic dipole moments. The excited magnetic moment would generate a magnetic field that opposes the applied field when its phase is reversed, if the frequency of the applied magnetic field is higher than the magnetic resonant frequency of the coupled strips. It could give rise to $\mu<0$ when the diamagnetic response is strong enough. Second, the intensity of resonant currents is distributed at different positions when the sizes of the geometrical dimensions are adjusted. As illustrated in Fig. 4(a), the resonant currents at $481 \mathrm{THz}$ mainly appear around the largest central holes. These currents gradually shift to smaller holes away from the center with enhanced frequency, which are listed in Figs. 4(b) and $4(\mathrm{c})$. The currents move to the smallest holes at the marginal positions, when the working frequency reaches the highest value of $529 \mathrm{THz}$. Thus, the resonant frequencies are strongly dependent on the featured sizes of the interior structure of 
the upper layer $481 \mathrm{THz}$ the lower layer

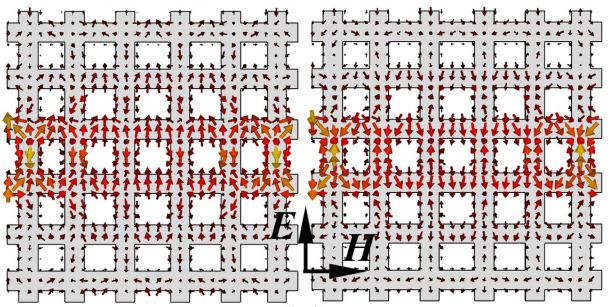

(a)

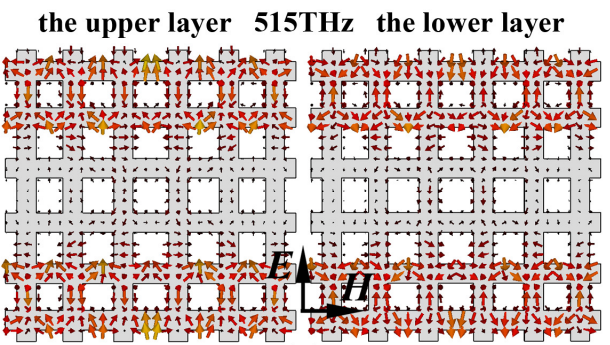

(c)

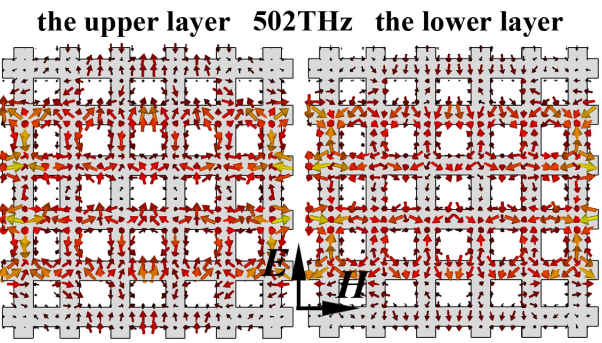

(b)

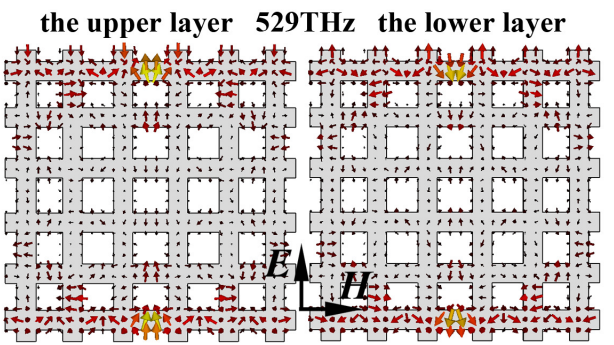

(d)

Fig. 4. The intensity distributions of resonant currents in the upper and lower silver layers at different frequencies: (a) $481 \mathrm{THz}$, (b) $502 \mathrm{THz}$, (c) $515 \mathrm{THz}$ and (d) $529 \mathrm{THz}$.

metamaterials. The characteristics of electromagnetic responses versus frequencies agree well with the effective medium theory.

For comparison, additional simulations of the conventional DF metamaterial with uniform holes were performed. In this case, all holes have the same size with $a_{1}=a_{2}=a_{3}=a_{4}=145 \mathrm{~nm}$, while the other structural parameters and boundary conditions are kept constant. For a brief contrast, only the magnitude of $\mathrm{S}$ parameters and the distributions of resonant currents are shown in Figs. 5(a)

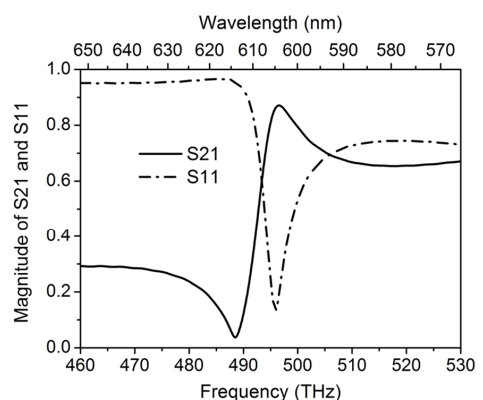

(a)

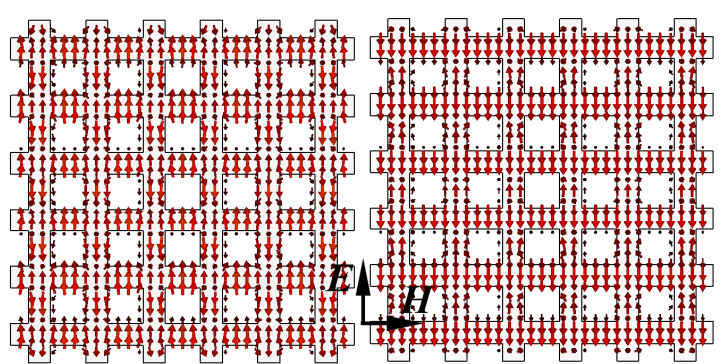

(b)

Fig. 5. Simulation results of the conventional DF metamaterial with the same hole size of $a_{1}=$ $a_{2}=a_{3}=a_{4}=145 \mathrm{~nm}$, while the other geometric parameters are kept the same with that of the model in Fig. 1. The calculated results are as follows: (a) magnitude of S21 and S11, (b) the currents distributions in the upper and lower silver layers at the resonant frequency of $489 \mathrm{THz}$. 
and 5(b), respectively. Only one transmission value is observed at $489 \mathrm{THz}$, at which the resonant currents in the upper and lower layers of silver are distributed uniformly and symmetrically. Thus, this result indirectly verifies the rationality and novelty of the new designed NIM.

\section{Further Design of a Multi-band Visible Absorber}

A low-profile nanostructured absorber modified from the new NIM can be obtained by substituting one perforated silver layer with a continuous silver film with the same thickness. Numerical results confirm that the absorber composed of silver mesh-PVA-silver film can achieve the multi-band absorption at visible frequencies. All descriptions of geometrical dimensions and boundary conditions are held similar to those in Fig. 1, but here $t=45 \mathrm{~nm}, h=30 \mathrm{~nm}, w=200 \mathrm{~nm}, a_{1}=457 \mathrm{~nm}$, $a_{2}=447 \mathrm{~nm}, \overline{a_{3}}=427 \mathrm{~nm}$, and $a_{4}=407 \mathrm{~nm}$. The electromagnetic wave is incident normally to the silver mesh side, and the simulated results are displayed in Fig. 6. In Fig. 6(a), the coefficients of transmission and reflection are expressed with percentage. Figure 6(b) illustrates the absorption which is attained from the expression of $(1-$ transmission - relection $) \times 100 \%$. Transmission approaches zero from $505 \mathrm{THz}$ to $535 \mathrm{THz}$. Meanwhile, the reflection also drops to zero at 511 , 520, 523, 525, and $527 \mathrm{THz}$. Finally, multi-band high absorptions in excess of $95 \%$ appear at the five frequencies, thereby effectively extending the working bandwidth.

Such high absorption results from the electromagnetic resonance between the upper layer of the silver mesh and the lower layer of the silver film. The bottom silver film is thick enough such that the incident wave cannot pass through it, and the resonances are sufficiently intense to dissipate the large energy, so leading to a small reflection. The causation of multi-band absorption can be discussed similar to the multi-band negative-index behavior. Owing to space limitation, we provide only the intensity distributions of resonant currents at the first resonant frequency
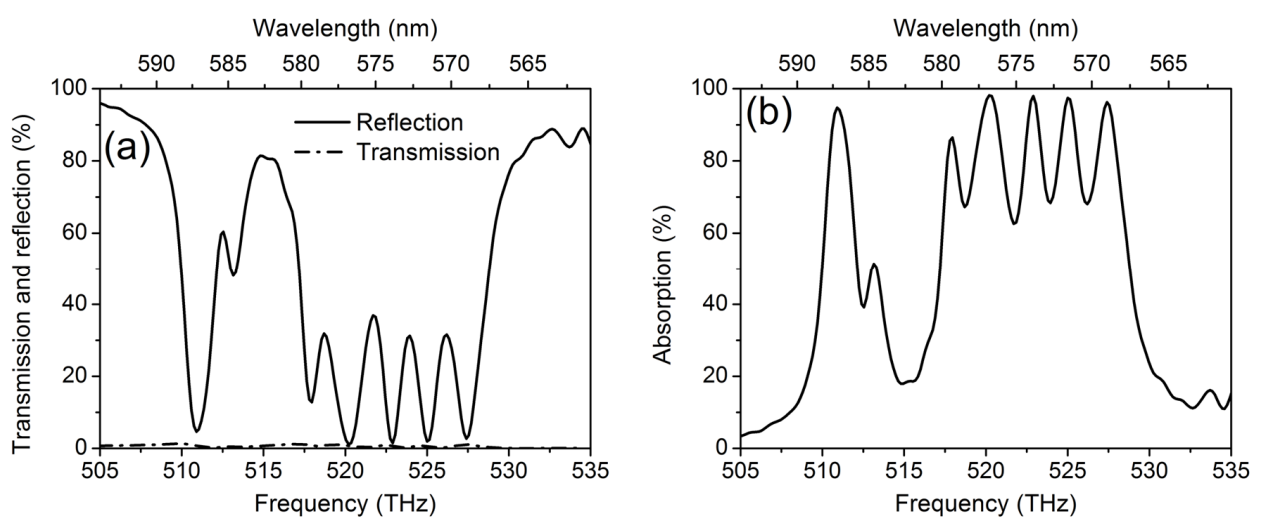

Fig. 6. Calculated results of the absorber composed of silver mesh-PVA-silver film which is transformed from the NIM model in Fig. 1. (a) Transmission and reflection. (b) Absorption. 


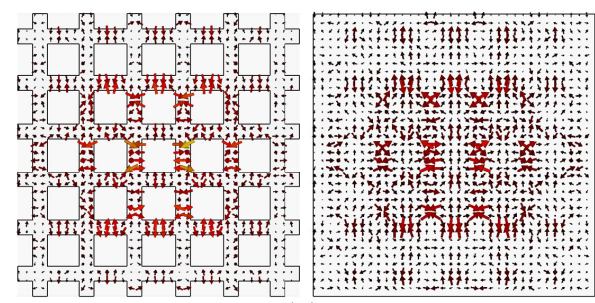

(a)

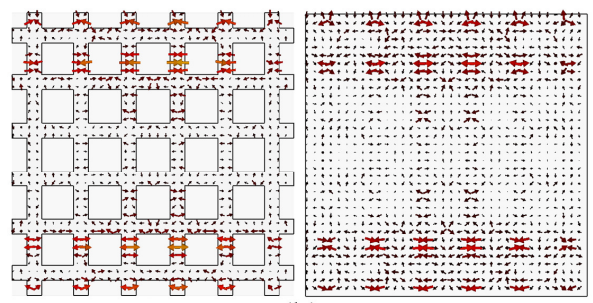

(b)

Fig. 7. Distributions of resonant currents in the upper layer of silver mesh and the lower layer of silver film at resonant frequencies of (a) $511 \mathrm{THz}$ and (b) $527 \mathrm{THz}$.

of $511 \mathrm{THz}$ and the last one of $527 \mathrm{THz}$, which are given in Figs. 7(a) and 7(b), respectively. In the same way, the absorption at lower frequencies is associated with the response of the central holes with larger sizes. By contrast, absorption at higher frequencies is related with the response of marginal holes with smaller sizes. Thus, the explanations for the working characteristics in Fig. 7 are consistent with those in Fig. 4.

\section{Conclusions}

Numerical results show that the new metamaterial with 16 different hole sizes could achieve multi-band negative index in a wide spectral region from $470 \mathrm{THz}$ to $540 \mathrm{THz}$. The physical mechanism was analyzed by discussing the relation between the distributions of resonant currents and structural sizes. Resonances at lower frequencies occurred at larger holes, whereas resonances at higher frequencies were observed at smaller ones. Several resonances appeared at adjacent frequencies across a wide range when the structural parameters were properly adjusted and optimized, which effectively broadened the operating band. In addition, a lowprofile nanostructured absorber was designed by replacing one layer of silver mesh with a silver film with the same thickness. This absorber could achieve multi-band absorption of more than $95 \%$ around visible frequencies of 511, 520, 523, 525 and $527 \mathrm{THz}$.

\section{Acknowledgment}

We acknowledge partial support from Northwestern Polytechnical University Scientific Research Allowance (No. G2015KY0302).

\section{References}

1. R. A. Shelby, D. R. Smith and S. Schultz, Science 292 (2001) 77.

2. N.-H. Shen, T. Koschny, M. Kafesaki and C. M. Soukoulis, Appl. Phys. Lett. 102 (2013) 241915.

3. X. Zhang and Z. Liu, Nat. Mater. 7 (2008) 435. 
4. C. Sabah, Mod. Phys. Lett. B 30(11) (2016) 1650186.

5. J. Chen, Y. Wang, B. Jia, T. Geng, X. Li, L. Feng, W. Qian, B. Liang, X. Zhang, M. Gu and S. Zhuang, Nat. Photonics 5 (2011) 239.

6. T. Ergin, N. Stenger, P. Brenner, J. B. Pendry and M. Wegener, Science 328 (2010) 337.

7. T. Li, M. Huang, J. Yang, X. Xu and M. Chen, Mod. Phys. Lett. B 29(11) (2015) 1550045.

8. F. Dincer, M. Karaaslan, E. Unal, O. Akgol and C. Sabah, Mod. Phys. Lett. B 29(18) (2015) 1550087.

9. Y.-T. Jin, L.-M. Si, Q.-L. Zhang, Y.-M. Wu and X. Lv, Mod. Phys. Lett. B 30(7) (2016) 1650079.

10. A. A. Houck, J. B. Brock and I. L. Chuang, Phys. Rev. Lett. 90(13) (2003) 137401.

11. S. Zhang, W. Fan, K. J. Malloy, S. R. J. Brueck, N. C. Panoiu and R. M. Osgood, J. Opt. Soc. Am. B 23(3) (2006) 434.

12. S. Xiao, V. P. Drachev, A. V. Kildishev, X. Ni and U. K. Chettiar, Nature 466 (2010) 735 .

13. C. García-Meca, Mod. Phys. Lett. B 27(15) (2013) 1330011.

14. B. Y. Gong, X. P. Zhao, Z. Z. Pan, S. Li, X. N. Wang, Y. Zhao and C. R. Luo, Sci. Rep. 4 (2014) 4713.

15. M. Navarro-Cía, C. García-Meca, M. Beruete, A. Martínez and M. Sorolla, Opt. Lett. 36(21) (2011) 4245.

16. K. Song, Q. Fu and X. Zhao, Phys. Scripta 84 (2011) 035402.

17. C. Sabah and H. G. Roskos, Curr. Appl. Phys. 12 (2012) 443.

18. A. C. Atre, A. García-Etxarri, H. Alaeian and J. A. Dionne, Adv. Opt. Mater. 1 (2013) 327.

19. Z.-C. Xu, R.-M. Gao, C.-F. Ding, Y.-T. Zhang, L. Wu, D.-G. Xu and J.-Q. Yao, Mod. Phys. Lett. B 29(12) (2015) 1550056.

20. J. Yang, C. Sauvan, H. T. Liu and P. Lalanne, Phys. Rev. Lett. 107 (2011) 043903.

21. D. Tanasković, M. Obradov, O. Jakšić and Z. Jakšić, Phys. Scripta T 162 (2014) 014048.

22. L. Gao, Y. Kim, A. Vazquez-Guardado, D. Franklin, C. J. Progler, G. R. Bogart, J. A. Rogers and D. Chanda, Adv. Opt. Mater. 2 (2014) 256.

23. G. Dolling, M. Wegener, C. M. Soukoulis and S. Linden, Opt. Express 15 (2007) 11536.

24. T. Koschny, M. Kafesaki, E. N. Economou and C. M. Soukoulis, Phys. Rev. Lett. 93 (2004) 107402.

25. D. R. Smith, D. C. Vier, Th. Koschny and C. M. Soukoulis, Phys. Rev. E 71 (2005) 036617. 